\title{
Emerging drugs for cancer-related pain
}

\author{
Sebastiano Mercadante
}

Received: 3 May 2011 / Accepted: 13 September 2011 /Published online: 23 September 2011

(C) Springer-Verlag 2011

\section{Introduction}

Most cancer pain can be managed effectively using analgesics according to World Health Organization (WHO) guidelines. This involves a stepwise approach to the use of analgesic drugs. While the validity of the WHO approach has been questioned, the 3-step ladder has been an invaluable tool and has been extensively validated $[25,37,41]$. This standardized approach is a framework of principles and allows flexibility in the choice of analgesics. A substantial minority of patients treated using the WHO ladder do not have a successful outcome because of excessive adverse effects, inadequate analgesia, or a combination of both adverse effects and inadequate analgesia [5]. Drug development in the area of cancer pain has been difficult owing to several reasons. Clinical research is a very difficult issue due to the characteristics of patients who are often unable to participate in long-term studies, because they are unstable. Recently animal models have been developed to mimic cancer pain. They have produced relevant information on the physiopathology, but limited data are available in clinical practice. However, there are new therapeutic strategies and a variety

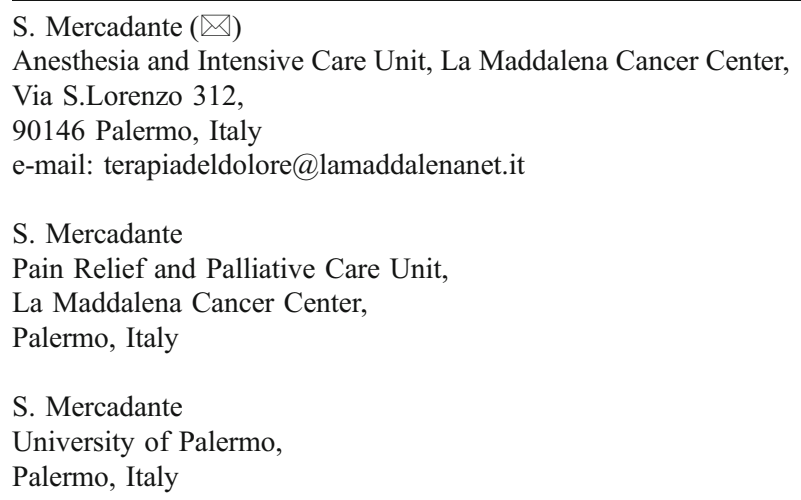

of novel drugs, including opioids and non-opioid drugs, that are based on established concepts with the goal to improve analgesia in patients with cancer-related pain.

\section{Medical need}

In the USA, cancer is second only to cardiovascular disease as the leading cause of death; in the UK, it is the leading cause of death. In many Third World countries, cancer incidence appears much lower, most likely because of the higher death rates due to infectious disease or injury. In the Eastern Mediterranean Region, cancer incidence is expected to increase by $100 \%$ to $180 \%$ in the next 15 years due to increases in life expectancy, an increasing proportion of elderly people, more risk factors, and the successful control of childhood disease. Cancer is responsible for about $25 \%$ of all deaths in the USA and is a major public health problem in many parts of the world [18].

Pain control is an important issue in cancer. The prevalence of chronic pain is about $30-50 \%$ among patients with cancer who are undergoing active treatment for a solid tumor and $70-90 \%$ among those with advanced disease. Pain is consistently one of the most feared consequences of cancer for both patients and families [5]. Although availability of guidelines and accumulating experience have greatly improved the possibility of satisfactory pain control for most patients with advanced cancer, pain remains less than optimally controlled. While some patients are often undertreated (due to physicians' poor knowledge and negative attitudes in pain management, and patient-related barriers), a consistent number of patients may not respond optimally to available analgesic therapies, due to complex mechanisms underlying pain syndromes as well as other individual factors. 


\section{Existing treatments}

Most pain in cancer responds to pharmacological management using orally administered analgesics. Current treatments are based on the analgesic ladder which involves a stepwise approach to the use of analgesic drugs and is essentially a framework of principles rather than a rigid protocol. The feasibility and efficacy of the analgesic ladder have been reported in different studies [25, 37, 41].

NSAIDs and acetaminophen are agents recommended in the WHO analgesic ladder and are suggested as the first step of the WHO analgesic ladder. This class of drugs has been found effective for mild cancer pain. A Cochrane Database Systemic Review of NSAIDs for treating cancer pain was not able to find clear evidence supporting use of one NSAID agent over another

\section{Opioids}

Opioids remain the most effective analgesics for moderate to severe pain and the mainstay of cancer pain management. They are regularly administered around the clock, providing extra doses for the treatment of pain exacerbation, currently defined as breakthrough pain. Despite available treatments and the development of effective guidelines for the management of cancer pain (WHO analgesic ladder), a large proportion of cancer patients remain undertreated due to barriers related to health care resources, patient, and family. Lack of adherence with analgesic regimen is a further barrier to effective cancer pain management. Misconceptions about opioids, insufficient education of healthcare professionals, and restrictive regulation in some countries for many years have been the main cause of the lack of application of simple guidelines of the WHO for cancer pain relief [26]. As the treatment should start at the step of the analgesic ladder appropriate for the severity of pain, all patients with moderate to severe pain should receive a trial of opioid analgesics, regardless of etiology.

Oral morphine has been widely used for treating pain of moderate to severe intensity and remains the opioid of choice for its familiarity, availability, and costs [14]. However, some patients may not respond to increasing doses of morphine, due to adverse effects before achieving an acceptable analgesia or poor analgesic response despite rapid dose escalation. Moreover, oral morphine is unable to provide prompt analgesia for the treatment of breakthrough pain, which requires drugs with a rapid onset to counteract the temporal profile of the pain flare.

It is now recognized that individual patients vary greatly in their response to different opioids. Patients who obtain poor response to one opioid will frequently tolerate another opioid. Several opioids are now available, although not in all the countries. Sequential opioid trials, also opioid rotation, or opioid switching may be needed to identify the drug that yields the most favorable balance between analgesia and adverse effects [27]. However, in some patients, the response may still result disadvantageous, as adverse effects may prevail on analgesia.

Despite the fact that cancer pain can be controlled by the regular use of opioids for chronic background pain, transitory exacerbations of pain in patients with relatively stable and adequately controlled background pain may consistently reduce the level of quality of life. Breakthrough cancer pain can be experienced several times per day and is characterized by a fast onset, usually reaching a peak of intensity within $3 \mathrm{~min}$, is often very severe, with an average duration of 30-60 $\mathrm{min}$ [28]. Cancer pain can be controlled by regular use of opioids for chronic background pain, but transitory pain may consistently reduce patients' quality of life. In addition to the regular opioid regimen given around the clock, breakthrough pain requires supplemental short-acting medication that should closely match the dynamics of pain episodes, with fast onset of action and short duration of effect. The treatment with conventional analgesics such as oral formulation of morphine-like drugs is clinically far from optimal for most patients because onset of pain relief usually occurs $30 \mathrm{~min}$ or more, when the episode may spontaneously disappear. Non-invasive methods with innovative delivery systems have been developed to meet the need for a rapid and potent analgesia.

\section{Non-opioid drugs}

The widely used adjuvant analgesics are an important part of the neuropathic pain armamentarium [23]; the choice of adjuvant analgesic is not based on potency superiority as all have a number needed to treat of about three [3].

Currently gabapentin and pregabalin are commonly used as adjuvant analgesics. To date, they have not been shown to have a lower number needed to treat than the older anticonvulsants. However, the lack of pharmacological interaction and general tolerability favor their use in advanced cancer patients receiving other drugs, including opioids [19]. The pharmacological action of the agents involve modulating specific voltage-gated calcium channels by binding to its $\alpha 2-\delta-1$ subunit. In comparison with gabapentin, pregabalin has a more predictable absorption profile including linear pharmacokinetics across its therapeutic dose range. As opposed to non-cancer pain states, few studies have evaluated gabapentin for the treatment of cancer-related pain. These studies showed significant differences in pain intensities, although the clinical meaning remains uncertain $[4,34]$. There have been no head-to-head studies to directly compare the efficacies of gabapentin and pregabalin. 
The most studied adjuvant agents are antidepressants. Tricyclic antidepressants have been recommended among first-line agents for neuropathic pain in non-cancer patients. The analgesic effects are likely because of enhancement of the endogenous, descending antinociceptive system, that is, inhibition of the reuptake of norepinephrine and serotonin. Overall, antidepressants have good efficacy but also have a heavy adverse-effects burden. Alternately, duloxetine is often used because of its efficacy comparable to tricyclics without the concomitant adverseeffects burden, as it is less sedative. However, studies of antidepressant agents for treatment of cancer pain are few in number [23].

\section{Current research goals}

There have been many important improvements in cancer pain management. Research into new drugs for the treatment of cancer pain falls into two broad categories: the development of non-opioid drugs with different mechanisms of action which may add further analgesia and improve the global analgesic response when added to opioids and the refinement in the use of current opioids through the development of new drugs, new delivery systems, and new combinations.

Despite proven analgesic efficacy, opioid use is associated with numerous and severe, unwanted adverse drug reactions, particularly opioid-induced bowel dysfunction that seriously impacts patients' quality of life. While many opioid side effects occur at the beginning of pain treatment and are attenuated or disappear over time, constipation and related symptoms persist or can even become worse. Agents used at present to manage opioid-induced constipation do not address the underlying opioid receptor-mediated cause of bowel dysfunction and are often ineffective. There is, therefore, a significant need for more effective treatment options.

Perhaps the most notable advancement in pain medicine is those emerging new drug-delivery systems that have extended the use of analgesics beyond conventional drugdelivery routes. In many cases, these new drug-delivery systems provide more comfort for patients through fewer adverse effects and a quicker onset of pain relief. There is a significant expansion of opioid analgesics along with innovative drug-delivery systems to meet the need for rapid analgesia, for example, for the management of breakthrough pain.

The list of non-opioid pain medication currently in clinical use has been expanded, although most of them have limited evidence in cancer pain management. Most of these new additions are those medications already in use for chronic non-cancer pain.

\section{New opioid formulations}

Several different drug-development strategies have attempted to reduce side effects by exploiting anatomic barriers to drug distribution.

Oxycodone-naloxone combination Prevention of opioidinduced constipation is considered to be a more effective strategy than merely treating it when it occurs. Laxatives do not address the opioid receptor-mediated mechanism of bowel dysfunction and do not provide sufficient relief. An approach to managing opioid-induced constipation without reversing the central analgesic action of opioids is to use opioid antagonists that are not systematically absorbed, such as naloxone, or which do not really penetrate the blood-brain barrier, such as methylnaltrexone.

A number of pioneer studies have assessed the ability of oral immediate release naloxone also showing some evidence for slightly reduced analgesic efficacy and intestinal withdrawal symptoms. A prolonged-release formulation could confer additional benefits ensuring continual antagonism and by reducing the risk of overburdening the hepatic enzymatic system responsible for its first-pass metabolism, thus reducing the risk of naloxone being systematically available with consequent loss of analgesic efficacy. A slow-release oxycodone-naloxone combination in a single formulation tablet has been assessed in noncancer patients with chronic pain provided effective pain relief with a reduction in opioid-induced constipation as well as the use of laxatives. The optimal ratio was 2:1. Only a minimal number of patients had cancer pain [24]. Thus, it is important to have more data in the cancer population who are at higher risk of constipation due to the large number of contributing factors [6].

A peripheral opioid antagonist, methylnaltrexone, has been recently developed as a novel therapy for opioidinduced constipation. The addition of a methylgroup reduces lipid solubility and diminishes the ability of the molecule to cross the blood-brain barrier. With these characteristics, methylnaltrexone has the potential to block opioid actions mediated by peripheral opioid receptors while sparing actions mediated by centrally located receptors. Different controlled studies have shown that methylnaltrexone promptly and predictably induced laxation in cancer patients with opioid-induced constipation. Bowel movements were observed in about half of patients within $4 \mathrm{~h}$ of administration, independent of age and opioid doses $[30,36]$.

Tapentadol Evaluation of a combined mechanism of action, $\mu$-opioid receptor activation with norepinephrine reuptake inhibition, has been undertaken to improve the therapeutic usefulness of opioid analgesics. Agents with the latter 
properties have been found to be particularly effective in neuropathic pain conditions and to potentiate the analgesic effects of opioids. Tapentadol is a centrally acting oral analgesic which possesses both mechanisms of action and has been shown to be efficacious in acute and chronic pain of diverse etiologies. Tapentadol has been reported to have similar efficacy compared with oxycodone, but a better gastrointestinal tolerability [39]. Possible advantages rely on less addiction potential and drug-drug interactions with agents affected by CYP activity or plasma protein binding. However, long-term studies in cancer patients with chronic pain are lacking.

\section{New delivery systems}

Rapid-onset opioids for breakthrough pain Breakthrough cancer pain can be defined as an acute transient worsening of pain. The management of breakthrough pain requires drugs with a rapid onset, which cannot be achieved with oral morphine. Alternative delivery systems have been developed in the recent years. Fentanyl is a highly potent opioid, enabling it to effectively treat the severe pain intensity pain that is characteristic of breakthrough cancer pain. Fentanyl can be administered by a number of different routes, including transmucosal and sublingual. Oral transmucosal fentanyl (OTFC) has been the first product developed for these purposes. Each OTFC unit consists of a lozenge containing fentanyl on a handle. The lozenge is applied against the buccal mucosa, and as it dissolves in saliva, a proportion of the drug diffuses across the oral mucosa. The rest is swallowed and partially absorbed in the stomach and intestine. Several studies have shown the superiority of OTFC over placebo and oral morphine in the treatment of breakthrough cancer pain [7]. However, OTFC requires active patient's collaboration, and patients may prefer "one shot" treatment, although less effective, like oral morphine [8].

The fentanyl buccal tablet (FBT) incorporates a novel drug-delivery platform, OraVescent technology, which employs an effervescent reaction to enhance fentanyl absorption through the buccal mucosa and facilitate rapid systemic exposure to the analgesic. Transient $\mathrm{pH}$ changes accompany the effervescence reaction and increase both the rate of tablet dissolution and membrane penetration of fentanyl. Availability of FBT was $48 \%$, in comparison with $22 \%$ of OTFC. Controlled studies have shown that FBT is effective in treating breakthrough cancer pain [32].

A new sublingual formulation of fentanyl has been developed. This takes the form of a small tablet that is based on a mix of water-soluble carrier particles coated with a mucoadhesive agent and active drug particles [20].
The purpose of the bioadhesive agent is to prolong the retention of the active drug particles on the oral mucosa to increase site-specific absorption of fentanyl across the oral mucosa, thereby reducing the risk of swallowing the drug. In cancer patients, sublingual fentanyl, compared to placebo, provided rapid and effective analgesia for the treatment of BTcP and was tolerated over 12 months of treatment [33].

The intranasal administration route is an alternative with several benefits, including fast systemic penetration and potential for self-administration, as well as acceptable to patients with dysphagia, nausea and vomiting, impaired gastrointestinal function, or salivary dysfunction. Reduced salivary flow and/or impaired function have been reported in more than $75 \%$ of patients with late-stage cancer. Different doses of intranasal fentanyl (INFS) showed a short $T_{\max }$, in the range of $12-15 \min$ [16]. In a randomized-controlled study, significantly more patients attained faster "meaningful" pain relief with INFS than OTFC, and more patients preferred INFS to OTFC. Pain intensity difference was significantly greater for INFS than OTFC from $5 \mathrm{~min}$ post-dosing, and clinically important pain relief (33\% reduction in pain intensity) was seen $5 \mathrm{~min}$ after INFS treatment in a quarter of breakthrough pain episodes. Furthermore, INFS was easy to use, with the majority of patients preferring INFS over OTFC [29]. INFS showed a greater efficacy over competing rapid-onset opioid formulations [38].

\section{Adjuvants}

For pain clinicians, the majority of cases where adjuvants are employed are for the treatment of neuropathic pain. Cancer-related neuropathic pain, which involves peripheral or central lesions of the nervous system, is typically a result of regional nerve damage from tumor infiltration into nerves and plexuses, radiation fibrosis, chemotoxicity, or surgical injury [31]. A concomitant treatment with adjuvant drugs may improve analgesia in patients with cancer pain, allowing less consumption of opioids and as a consequence a better tolerability of the analgesic treatment. A different mechanism of action may provide further analgesia when added to opioids. Antidepressants and anticonvulsants are the most well-studied drug classes for neuropathic cancer-related pain. Currently, gabapentin and pregabalin among anticonvulsants are commonly used as adjuvant analgesics. To date, they have not been shown to have a lower number needed to treat than the older anticonvulsants. Despite the evidence-based efficacy demonstrated in non-cancer pain conditions, no large experience exists with antidepressant drugs in cancer patients. 
Newer antidepressants have been developed, such as venlafaxine and duloxetine, but no study has been performed in cancer patients.

Cannabinoids Cannabinoids act primarily through specific receptors: $\mathrm{CB} 1$ receptors are predominantly distributed in the central nervous system, and CB2 receptors are located primarily in the periphery (including the immune system) [22]. In cancer pain, cannabinoids have demonstrated efficacy comparable to some specific opioids like codeine [23]. There is evidence of synergy between cannabinoids and morphine, cannabinoids modulating endogenous opioid tone. Sativex is a combination of delta-9-tetrahydrocannabinol (THC) and cannabidiol (CBD) and has been developed for the relief of neuropathic pain in multiple sclerosis and the persistent background cancer-related pain. In a recent study, THC-CBD was more effective than placebo and THC alone in patients who experienced inadequate analgesia despite chronic opioid therapy. However, THC-CBD produced a consistent impairment of cognitive function. The study medication was delivered using a pump action oromucosal spray, and patients self-titrated to their optimal dose on an asneeded basis [18]. No precise doses were indicated. Thus, it is necessary to have more information to justify the use in cancer patients receiving opioid drugs for their pain

Ziconotide Intrathecal delivery of systemic analgesics is often used for cancer patients unresponsive to systemic therapy. Ziconotide has been approved by the FDA for severe chronic pain in patients who have failed other options. Ziconotide is an N-type calcium channel antagonist which reduces neurotransmitter release in the dorsal horn of the spinal cord. In a randomized-controlled, double-blind trial of ziconotide versus placebo in patients with refractory pain from cancer or AIDS, a significant improvement in pain intensity scores has been found [35]. However, ziconotide has been regarded as a difficult drug for the need of a slow titration and has a black box warning indicating a potential for neurologic impairment and psychiatric symptoms [12]. In cancer pain, the available evidence of the efficacy of intrathecal ziconotide after an unsuccessful treatment with systemic opioids is too weak in view of the potential risks.

Tetrodoxin Sodium channels are found on most nociceptive pain fibers. Tetrodoxin is a neurotoxin found in puffer fish and other marine animals and is a selective blocker of voltage-gated sodium channels, which mediate the depolarization phase of the action potential and are key regulators of neuronal excitability. Recent trials have suggested that tetrodotoxin, given for four consecutive days subcutaneously may potentially relieve moderate to severe pain for prolonged periods following treatment [13].
However, there was only a non-statistically significant trend toward more responders in the active treatment arm based on the primary endpoint, which was the pain intensity difference.

NMDA antagonists There is good evidence from experimental animal models, human volunteer studies, and small clinical trials that NMDA receptor antagonists relieve some types of neuropathic pain and may enhance the analgesic effects and prevent tolerance that may result from chronic opioid administration. Clinical reports indicate that ketamine in low doses as an adjuvant to opioid treatment may improve analgesia [2]. However, its use is restricted by unpleasant central adverse effects and requires some expertise. Dextromethorphan, a well-tolerated drug administered orally, has been used in combination with morphine. However, no clinical benefit has been found in patients with cancer pain [10]. Three trials failed to demonstrate enhanced opioid analgesia or reduction of tolerance when adding dextromethorphan to opioids in the treatment of chronic pain [11].

\section{Potential development issues}

Despite few success stories, extensive efforts are being made for new drug development, and there are many potential targets of new drug development which have been identified through preclinical research. Some of these targets have gone through extensive clinical trials. In general, most of these drugs need more safety information regarding their narrow therapeutic window, as they target different cellular sites of the nociceptive pathways, which often lack the specificity on the site of action [21].

New generations of drugs could be available soon. A novel N-type calcium channel blocker leconotide could have wider clinical applications than ziconotide. Unlike ziconotide, powerful anti-hyperalgesia without side effects can be achieved by intravenous administration of leconotide, thus avoiding the need for an intrathecal injection [17].

Ralfinamide has been demonstrated to have analgesic effects in animal models of somatic pain (formalin injection, inflammation induced by complete Freund's adjuvant and sciatic nerve ligation, as well as visceral pain). It seems likely that these effects contribute to the analgesic actions of ralfinamide. Suppression of neuropathic pain-related behavior was likely caused by a combination of mechanisms reported for ralfinamide, including inhibition of sodium and calcium currents, inhibition of substance $\mathrm{P}$ release, NMDA receptor antagonism, and neuroprotection [40].

JNJ-42160443 is a fully human recombinant monoclonal antibody that has the specific capacity to neutralize the 
biologic actions of the human nerve growth factor (NGF). NGF has been shown to contribute to persistent pain in a variety of animal models of inflammatory and neuropathic pain and is known to be elevated in many chronic painful conditions in humans. Anti-NGF therapy could be particularly effective in blocking cancer pain as NGF seems to be integrally involved in up-regulation, sensitization, and disinhibition of multiple neurotransmitters, ion channels, and receptors in the primary afferent nerve and dorsal root ganglia fibers that synergistically increase nociceptive signals originating from the tumor [15].

Osteoprogenin is a promising agent, which may act through reduction of osteoclast function to diminish tumorinduced bone destruction. This substance has been shown to suppress bone resorption as indicated by a rapid, sustained, and profound decrease of urinary N-telopeptide of collagen/ creatinine in different cancer models and may hold future promise [9].

Pharmacogenetics may very well play a critical role in personalizing pain management in the future. Recent research has identified and characterized the important roles that genetic variability plays in clinical opioid therapy outcomes. Polymorphisms within the genes encoding opioid receptors affect the opioid response. Moreover, polymorphisms in metabolic enzymes, as well as polymorphisms in transport proteins, affect opioid metabolism [1]. In the future, personalized opioid treatment could lead to a better selection of optimal therapy, fewer adverse effects, better adherence to therapy regimens, and reduced health care costs.

\section{Conclusion}

Cancer pain is a very complex issue which is challenging for clinicians and researchers. Animal models contributed to improve the knowledge on the principal mechanisms of cancer pain. While effective and tested treatments are available, new generations of drugs and delivery systems may allow to resolve some complex clinical situations improving analgesia with different mechanisms or decreasing the occurrence of adverse effects by limiting the use of high doses of opioids. While promising, information about new drugs is still limited, and more studies should support a clinical use in humans.

\section{Conflict of interest None.}

\section{References}

1. Argoff CE (2010) Clinical implications of opioid pharmacogenetics. Clin J Pain 26:S16-S20
2. Bell R, Eccleston C, Kalso E (2003) Ketamine as adjuvant to opioids for caner pain. A qualitative systematic review. Pain Symptom Manag 26:867-875

3. Bennett MI (2011) Effectiveness of antiepileptic or antidepressant drugs when added to opioids for cancer pain: systematic review. Palliat Med 25:553-559

4. Caraceni A, Zecca E, Bonezzi C et al (2004) Gabapentin for neuropathic cancer pain: a randomized controlled trial from a gabapentin. J Clin Oncol 22:2909-2917

5. Cherny N, Ripamonti C, Pereira J, Davis C, Fallon M, McQuay H, Mercadante S, Pasternak G, Ventafridda V (2001) Strategies to manage the adverse effects of oral morphine: an evidence-based report. J Clin Oncol 19:2542-2554

6. Clemens KE, Mikus G (2010) Combined oral prolonged-release oxycodone and naloxone in opioid-induced bowel dysfunction: review of efficacy and safety data in the treatment of patients experiencing chronic pain. Expert Opin Pharmacother 11:297-310

7. Davies A, Dickman A, Reid C, Stevens A, Zeppetella J (2009) The management of cancer related breakthrough pain: recommendations of a task group of the Association for Palliative Medicine of Great Britain and Ireland. Eur J Pain 13:330-337

8. Davies AN, Vriens J, Kennett A, McTaggart M (2008) An observational study of oncology patients' utilization of breakthrough pain medication. J Pain Symptom Manage 35:406-411

9. Delaney A, Fleetwood-Walker SM, Colvin LA, Fallon M (2008) Translational medicine: cancer pain mechanisms and management. Br J Anaesth 101:87-94

10. Dudgeon D, Bruera E, Gagnon B, Watanabe S, Allan S, Wart D, MacDonald S, Savage C, Tu D, Pater J (2007) A phase III randomized, double-blind, placebo-controlled study evaluating dextromethorphan plus slow-release morphine for chronic cancer pain relief in terminally ill patients. J Pain Symptom Manage 33:365-371

11. Galer B, Lee D, Ma T, Nagle B, Schlagheck T (2005) Morphidex (morphine sulphate/dextromethorphan hydrobromide combination) in the treatment of chronic pain: three multicenter, randomized, double-blind, controlled clinical trials fail to demonstrate enhanced opioid analgesia or reduction in tolerance. Pain 115:284-295

12. Gordon DB, Dahl JL, Miaskowski C et al (2005) American Pain Society recommendations for improving the quality of acute and cancer pain management. American Pain Society Quality of Care Task Force. Arch Intern Med 165:1574-1580

13. Hagen N, du Souich P, Lapointe B, Ong-Lam M, Dubuc B, Walde D, Love R, Ho NA (2008) Tetrodotoxin for moderate to severe cancer pain: a randomized, double blind, parallel design multicenter study. J Pain Symptom Manag 35:420-429

14. Hanks GW, Expert Working group of the Research Network of the European Association for Palliative Care (2001) Morphine and alternative opioids in cancer pain: the EAPC recommendations. $\mathrm{Br}$ J Cancer 84:587-593

15. Helfi FF, Rosenthal A, Walicke PA et al (2006) Novel class of pain drugs based on antagonism of NGF. Trend Pharm Sci 27:1-7

16. Kaasa S, Moksnes K, Nolte T, Lefebvre-Kuntz D, Popper L, Kress HG (2010) Pharmacokinetics of intransal fentanyl spray in patients with cancer and breakthrough pain. J Opioid Manag 6:17-25

17. Jemal A, Siegel R, Ward E et al (2008) Cancer statistics 2008. CA Cancer J Clin 58:71-96

18. Johnson J, Burnell-Nugent M, Lossignol D, Ganae-Motan ED, Potts R, Fallon MT (2010) Multicenter, double-blind, randomized, placebo-controlled, parallel-group study of the efficacy, safety, and tolerability of THC:CBD extract and THC extract in patients with intractable cancer-related pain. J Pain Symptom Manage 39:167-179

19. Laird B, Colvin L, Fallon M (2008) Management of cancer pain: basic principles and neuropathic cancer pain. Eur J Cancer 44:1078-1082 
20. Lennernas B, Frank-Lissbrant I, Lennernas H, Kalkner KM, Derrick R, Howel J (2010) Sublingual administration of fentanyl to cancer patients is an effective treatment for breakthrough pain: results from a randomized phase II study. Palliat Med 24:286-293

21. Mao J (2009) Translational pain research: achievements and challenges. J Pain 10:1001-1011

22. Martin-Sanchez E, Furukawa T, Taylor J, Martin JL (2009) Systematic review and meta-analysis of cannabis treatment for chronic pain. Pain Med 10:1353-1366

23. McGeeney BE (2008) Adjuvant agents in cancer pain. Clin J Pain 24(suppl):S14-S20

24. Meissner W, Leyendecker P, Mueller-Lissner S et al (2009) A randomized controlled trial with prolonged-release oral oxycodone and naloxone to prevent and reverse opioid-induced constipation. Eur J Pain 13:56-64

25. Mercadante S (1999) Treatment and outcome of cancer pain in advanced cancer patients followed at home. Cancer 85:1849-1858

26. Mercadante S (2007) Why are our patients still suffering? Clin Pract Nature Oncol 4:138-139

27. Mercadante S, Bruera E (2006) Opioid switching: a systematic and critical review. Cancer Treat Rev 32:304-315

28. Mercadante S, Radbruch L, Caraceni A, Cherny N, Kaasa S, Nauck F, Ripamonti C, De Conno F, Steering Committee of the European Association for Palliative Care (EAPC) Research Network (2002) Episodic (breakthrough) pain: consensus conference of an expert working group of the European Association for Palliative Care. Cancer 94:832-839

29. Mercadante S, Radbruch L, Davies A, Poulain P, Sittle T, Perkins P, Colberg T, Camba MA (2009) A comparison of intranasal fentanyl spray with oral transmucosal fentanyl citrate for the treatment of breakthrough cancer pain: an open-label, randomised, crossover trial. Curr Med Res Opin 11:2805-2815

30. Portenoy RK, Thomas J, Moehl BM (2008) Subcutaneous methylnaltrexone for the treqtment of opioid-induced constipation in patients with advanced illness: a double-blind, randomized, parallel group, dose-ranging study. J Pain Symptom Manage $35: 458-468$
31. Portenoy RK (1989) Cancer pain. Epidemiology and syndromes. Cancer 63:2298-2307

32. Portenoy RK, Taylor D, Messina J, Tremmel L (2006) A randomized, pladebo-controlled study of fentanyl buccal tablets for breakthrough pain in opioid-treated patients with cancer. Clin J Pain 22:805-811

33. Rauck R, Tark M, Reyes E, Hayes T, Bartkowiak A, Nalamachu S, Derrick R, Howell J (2009) Efficacy and long-term tolerbility of sublingual fentanyl orally disintegrating tablet in the treatment of breakthrough cancer pain. Curr Med Res Opin 25:2877-2885

34. Ross JR, Goller K, Hardy J et al (2005) Gabapentin is effective in the treatment of cancer-related neuropathic pain: a prospective, open-label study. J Palliat Med 8:1118-2116

35. Staats PS, Yearwood T, Charapata SG, Presley RW, Wallace MS, Byas-Smith M, Fisher R, Bryce DA, Mangieri EA, Luther RR, Mayo M, McGuire D, Ellis D (2008) Intrathecal ziconotide in the treatment of refractory pain in patients with cancer or AIDS: a randomized controlled trial. Pain Med 9:271-281

36. Thomas J, Karver S, Cooney GA et al (2008) A randomized placebo-controlled trial of subcutaneous methylnaltrexone for the treatment of opioid-induced constipation in patients with advanced illness. N Engl J Med 358:2332-2334

37. Ventafridda V, Tamburini M, Caraceni A, De Conno F, Naldi F (1987) A validation study of the WHO method for cancer pain relief. Cancer 59:850-856

38. Vissers D, Stam W, Nolte T, Lenre M, Jansen J (2010) Efficacy of intranasal fentanyl spray versus other opioids for breakthrough pain in cancer. Curr Med Res Opin 26:1037-1045

39. Wade W, Spruill WJ (2009) Tapentadol hydrochloride: a centrally acting oral analgesic. Clin Ther 31:2804-2818

40. Yamane H, de Groat WC, Sculptoreanu A (2007) Effects of ralfinamide, a $\mathrm{Na}^{+}$channel blocker, on firing properties of nociceptive dorsal root ganglion neurons of adult rats. Exp Neurol 208:63-72

41. Zech DFJ, Grond S, Lynch J, Hertel D, Lehmann KA (1995) Validation of World Health Organization guidelines for cancer pain relief: a 10-year prospective study. Pain 63:65-76 